\title{
Quality Control of PCB using Image Processing
}

\author{
Rasika R. Chavan \\ Gokhale Education Society's \\ R.H.Sapat College of \\ Engineering \\ Management and Research, \\ Nashik 05
}

\author{
Swati A. Chavan \\ Gokhale Education Society's \\ R.H.Sapat College of \\ Engineering \\ Management and Research, \\ Nashik 05
}

\author{
Gautami D. Dokhe \\ Gokhale Education Society's \\ R.H.Sapat College of \\ Engineering \\ Management and Research, \\ Nashik 05
}

\author{
Mayuri B. Wagh \\ Gokhale Education Society's \\ R.H.Sapat College of Engineering \\ Management and Research, \\ Nashik 05
}

\author{
Archana S.Vaidya \\ Gokhale Education Society's \\ R.H.Sapat College of Engineering \\ Management and Research, \\ Nashik 05
}

\begin{abstract}
An automated testing system for Printed Circuit Board (PCB) is preferred to get the technological advances in PCBs design and manufacturing, eliminates particular aspects and then provides fast, quantitative, and dimensional impositions. It reduces the testing time and manufacturing cost as human inspectors decisions are ineffective, slow and costly. Thus in this area, digital image processing can be used mainly for the detection of faulty parts or missing components.

This system mainly deals with analysis to detect faulty PCB. Digital camera is used in automated visual inspection system that captures image of each sample PCB product. The captured image is then provided to computer for further processing which includes conversion in various forms such as Gray scale image and binarized image. XOR operation is performed on these converted images to obtain the required results. Contour Analysis is performed on these results for classification. Missing components, polarities, circuit breaks, missing tracks these types of faults are detected and classified accordingly. This concept increases the speed and accuracy, eliminates human errors which are frequent in quality testing and also overcomes the weakness in the existing system. Hence the productivity can be increased by replacing manual testing with the proposed concept.
\end{abstract}

\section{General Terms}

Image Processing, Quality Control, Printed Circuit Board.

\section{Keywords}

Image Processing, Printed Circuit Board, Defect Detection, RGB, Gray Scale, Binarization, Edge Detection, Classification system.

\section{INTRODUCTION}

Now a days automated testing is necessary to improve the quality of PCB. In electronic industry there are defects, misalignment, and exposure error so automated testing is required. The defects can be detected by fault detection system using algorithms developed for it. So it improves the quality measures of $\mathrm{PCB}$ production and accuracy in the testing process. This system has advantage over manual testing in which exhaustion, slowness and high cost is involved [1]. In recent years, the PCB industries require automation due to many reasons. New electronic component fabrication techniques require efficient $\mathrm{PCB}$ design and testing method with proper dimension [2].Because of improper shape and size, PCB would not perform as required as design specification. The complex and compact design causes difficulties to manual testing process. Another important factor is necessity to reduce the testing time efficiently and reducing efforts. These factors lead to automation in PCB industry.

During the manufacturing there are some defects commonly found on PCB. These defects are divided into two types, potential and fatal defects. Short-circuit and open-circuit defects are fatal defects. Breakout, under etch, missing hole, and wrong size hole are potential defects. Fatal defects are those in which the PCB does not meet the purpose for which it is designed, while the potential defects are those which compromise the PCB performance during utilization.

\section{LITERATURE SURVEY}

In Industrial development and production, quality imposition and maintenance are growing in account. For the production of high quality final product, accurate specifications have to be met to ensure problem free assembly. Testing team in the industry strive to catch faults before the product is released but they always and they often reappear, even with the best manual testing process. Automated testing method is the best way to increase efficiency and analysis of your product testing [3]. Typical faults such as missing components as well as tracks, holes, circuit breaks can be found with Quality Control of PCB using Image Processing [4]. It also shows the divergence for a fast evaluation of fault detection. This means early detection of possible problems so that process can be corrected in time, resulting in efficient quality control. Industries that implement these automated testing techniques benefit for lower testing time for product inspection [5].

Sometimes, the defects in the components are found after the delivery of the product to the respective customers, even after effective manual testing. This leads to wastage of the product and manufacturing cost or requires rechecking. Component readings and values are checked and records are maintained in the computers. Companies remain competitive because they help their customer to go from idea to product faster, optimize production workflow and minimize rejects while production is running.

Previously designed systems are used only for detecting the faults such as broken tracks in bare Printed Circuit Boards. These techniques are not suitable to detect the faults on mounted PCBs. It cannot detect faults like short circuit, 
missing components and component polarities which are covered in proposed system. These systems are highly affected by lightning conditions [6].

From image processing article,2010 [7], PCB defects are classified into two categories functional defects and cosmetic defects. Functional defects affect the performance and quality of the PCB product where cosmetic defects affect the appearance of the PCB product which can be hazardous while heat dissipation and high voltage current. In this system, images are segmented and classify the defects into group of defects such as, square segment, hole segment, thick line segment, thin line segment. These defects are trained from the template image into segment. If other than this defect is occurred, it cannot detect the segment of defect. So that there is a limitation of detection of faults.

The system proposed by Takumi Uemura [8], is only applicable to grayscale image. In the further research, it can be applicable to color images or other images. They proposed the use of boundary code (BC) to detect the edges of the images. $\mathrm{BC}$ detects the edges as a virtual edges were set in virtual space between pixels. They will verify the application of proposed method to RGB images in future work.

\section{QUALITY VERIFICATION USING IMAGE PROCESSING}

To certify the quality of products typical Digital Image Processing techniques are hired. This is achieved by taking image of product PCB using high resolution camera. Further processing is done on captured image.

This proposed idea of using Digital Image Processing helps to save time of quality testing as the process becomes fully automated and results in improved quality. Human efforts are reduced in terms of testing time.

\section{ARCHITECTURAL DESIGN}

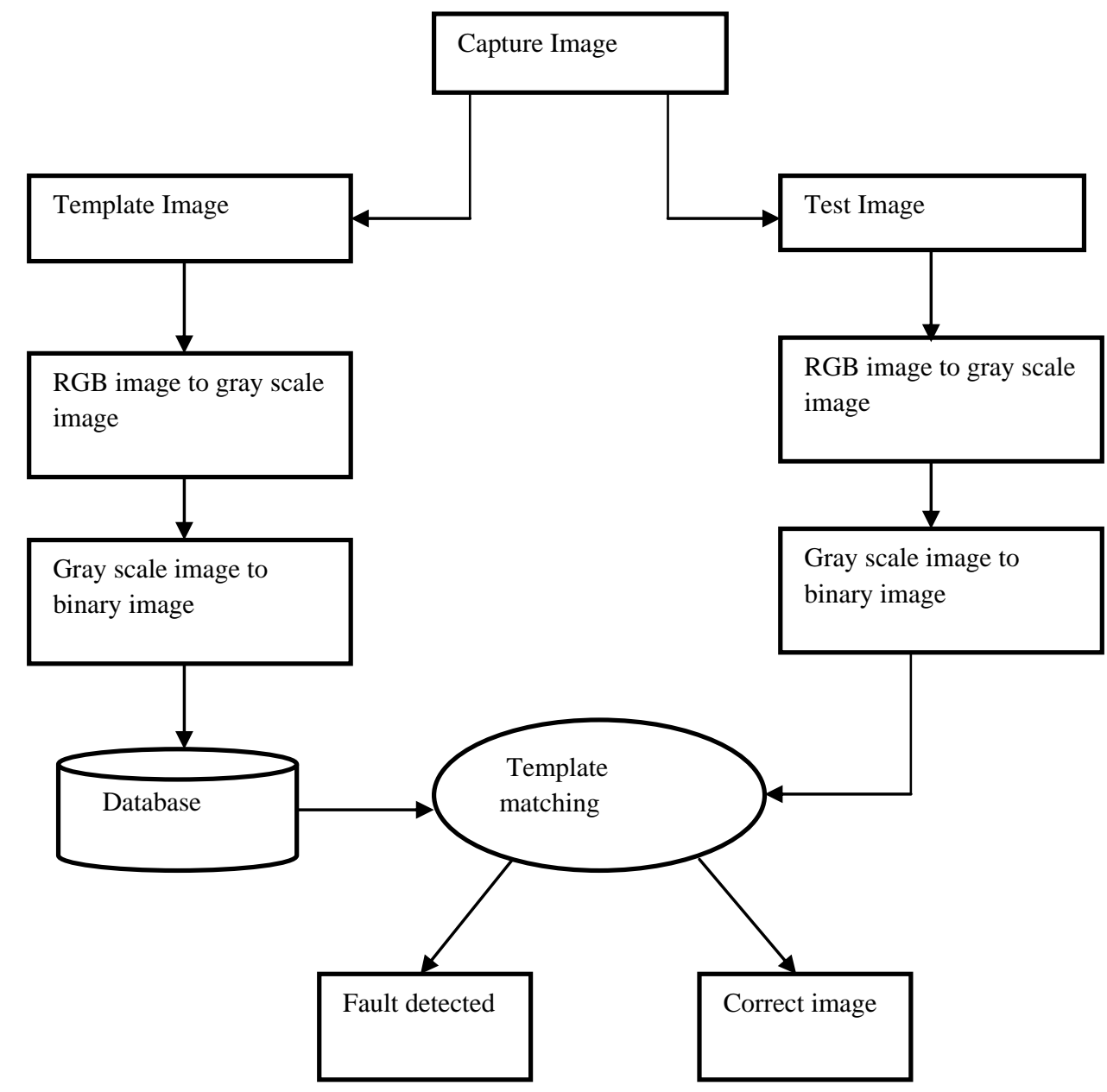

Fig 1: System Architecture

Fig1. shows the overall architecture of Fault Detection System. The product i.e., PCB is placed under the High Resolution Camera to obtain RGB image of PCB. The captured RGB image is then converted to gray scale. This gray scale image is the input for the binarization. Gray scale image is then converted to binary form using Canny Edge Detection algorithm. The template image is stored in the database. Every test image is compared with this template image. Template matching is done using XOR operation. Results of template matching is stored in the database.

\section{ALGORITHM}

Various algorithms are used in the proposed system such as Fault Detection Algorithm, Canny Edge Detection Algorithm and Contour Analysis. 


\subsection{Fault Detection Algorithm}

Input: Color images taken from high resolution camera of 13 Megapixel.

Output: PCB is inspected for faults and classified further into different categories.

\section{Start:}

Algorithm steps for image processing are as follows:

Step 1: The two dimensional RGB image is converted into grayscale image. Each of $\mathrm{R}, \mathrm{G}$ and $\mathrm{B}$ color pixel has a range of $[0,255]$. If all the three color pixels are merged together and have a same pixel value at corresponding coordinates, it results into grayscale image. It is calculate as,

$$
(\mathrm{R}+\mathrm{G}+\mathrm{B}) / 3
$$

Step 2: Grayscale image is subjected to get a binary image. Binary image is treated as black and white image in terms of 0 's and 1's.Set the threshold value for interpretation of the pixel value. If the pixel value is greater than threshold then set the pixel with the value 1 (white) and replaces all other pixel with 0(black).

Step 3: After the image conversion, apply bitwise XOR operation on two images which are in binary form to identify the defects. XOR operation is only true if one of the input value is true otherwise false.

Step 4: Contour Analysis technique is used to train the images of the PCB. It scans the whole image pixel by pixel and used for labeling of faults.

Step 5: Results are generated in terms of whether sample image is faulty or not.

\section{End}

\subsection{Canny Edge Detection Algorithm}

Canny Edge Detection algorithm [7][5] is mainly used for edge detection. It aims to satisfy three main criteria such as: Low error rate, Good localization and Minimal response.

Input: Gray scale image is taken as input

Output: Edges are detected in the binarized image.

\section{Start:}

The algorithm runs in following steps:

Step 1: Noise Reduction - First step is to remove noise from the image using Gaussian filter. Gaussian filter is a nonuniform low pass filter. It is used to blur the images. Resulting image is the smoothened image. Two dimensional Gaussian function is used when working with images. It is given by,

$G(x, y)=\frac{1}{2 \pi \sigma^{2}} e^{-\frac{x^{2}+y^{2}}{2 \sigma^{2}}}$

Where, $\mathrm{x}$ is the distance from origin in the horizontal axis, $\mathrm{y}$ is the distance from origin in the vertical axis and $\sigma$ is the standard deviation of the distribution. The distribution is assumed to have a mean of 0 .

Step 2: Find the intensity gradients of the image Smoothened image is then filtered with the sobel kernel. Apply the pair of convolution masks in $\mathrm{x}$ and $\mathrm{y}$ direction then finds the gradient strength and direction. The direction is rounded to one of the four angles $(0,45,90$ or 135$)$.

$$
\text { Edge_Gradient }(\mathrm{G})=\sqrt{G_{x}^{2}+G_{y}{ }^{2}}
$$

$$
\text { Angle }(\Theta)=\tan ^{-1}\left(\frac{G_{y}}{G_{x}}\right)
$$

Where, $\mathrm{Gx}$ is used to find intensity gradient along horizontal direction, Gy is used to find intensity gradient in vertical direction and angle $(\Theta)$ is used to calculate Gradient Direction.

The edges should be marked where the gradients of the image has large magnitudes.

Step 3: Non-maximum suppression - After getting gradient magnitude and direction, a full scan of image is done to remove any unwanted pixel which may not constitute the edge. For this, at every pixel, Pixel is checked if it is local maximum in its neighborhood in the direction of gradient.
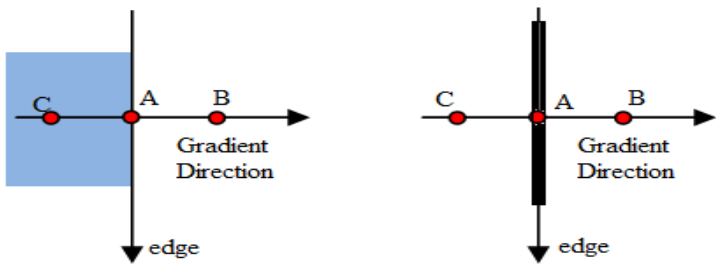

Fig 2: Non-maximum suppression

Point $\mathrm{A}$ is on the edge (in vertical direction). Gradient direction is normal to the edge. Point $\mathrm{B}$ and $\mathrm{C}$ are in gradient directions. So point A is checked with point $\mathrm{B}$ and $\mathrm{C}$ to see if it forms a local maximum. If so, it is considered for next stage, otherwise, it is suppressed (put to zero).

In short, the result you get is a binary image with "thin edges".

Step 4: Hysteresis Thresholding - Canny algorithm does use two thresholds (upper and lower) for determining which edges are really edges or which are not:

1. If the value of a pixel gradient is higher than the upper threshold, the pixel is considered as on edge.

2. If the value of a pixel gradient is below lower threshold, then it is rejected.

3. If the value pixel gradient is between the two thresholds, then it will be considered only if it is connected to a pixel that is above the upper threshold.

\subsection{Contour Analysis}

In the PCB fault detection system, we are using the Contour Analysis for image reorganization and labeling of faults by analyzing the type of faults.

The Contour Analysis allows describing, comparing, storing and finding the objects by the exterior outlines - contours. Contour is a boundary of object separating object from a background. It is assumed that the contour contains the necessary information of the object. Interior points of the object are not considered for processing. It restricts area of working of algorithm of a Contour Analysis. The contours allows to pass from only two-dimensional space of the image .Contour Analysis varies according to the pattern of the image and invariant to the transposition.[9]

In a Contour Analysis, the contour is recorded by the sequence of complex numbers. On a contour, the starting point of image is fixed. Then, the contour is scanned in clockwise manner, and each vector of offset is noted by a 
complex number $a+i b$. Where a-offset on $x$ axis, and $b$ offset on y axis. Offset is noted concerning the previous point. [11]

In the PCB fault detection system, Contour Analysis is specific to applicable area. On the binarized image, contours are selected and each part of the image is separate out. It can train the different template image to find the contours and searching and comparing of sample image with template image to find the maximum similar part of the image.

The Contour Analysis in the PCB fault detection system improves the speed and performance of the image .It clearly detects the fault area with labeling of faults by analyzing the type of faults accordingly.

\section{RESULTS AND DISCUSSTION}

\subsection{Reference Image}

This is the image taken of the PCB that is to be tested which is the input for fault detection system. Fig. 3 shows the reference image captured from sample PCB.

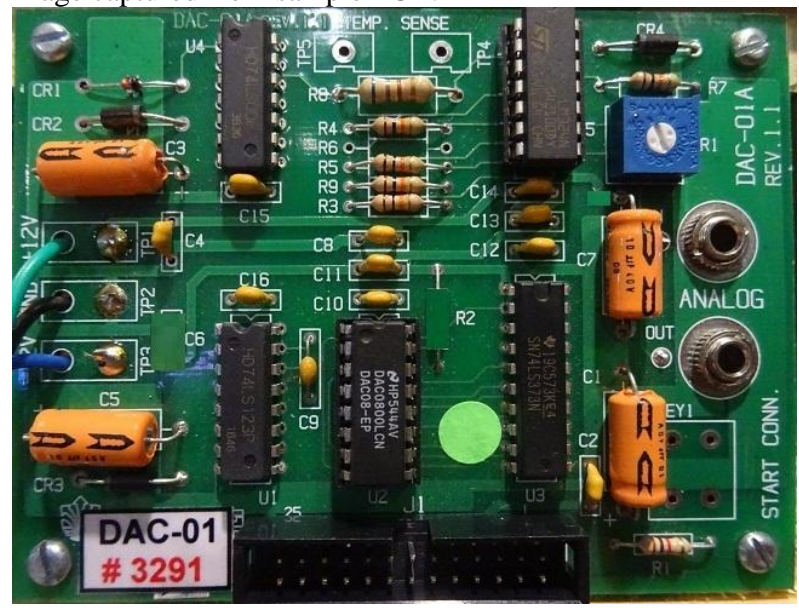

Fig 3: Reference Image

\subsection{Template Image}

This is the image of Standard template PCB which is used for comparison with the reference image. Fig.4 shows the image captured of standard template PCB.

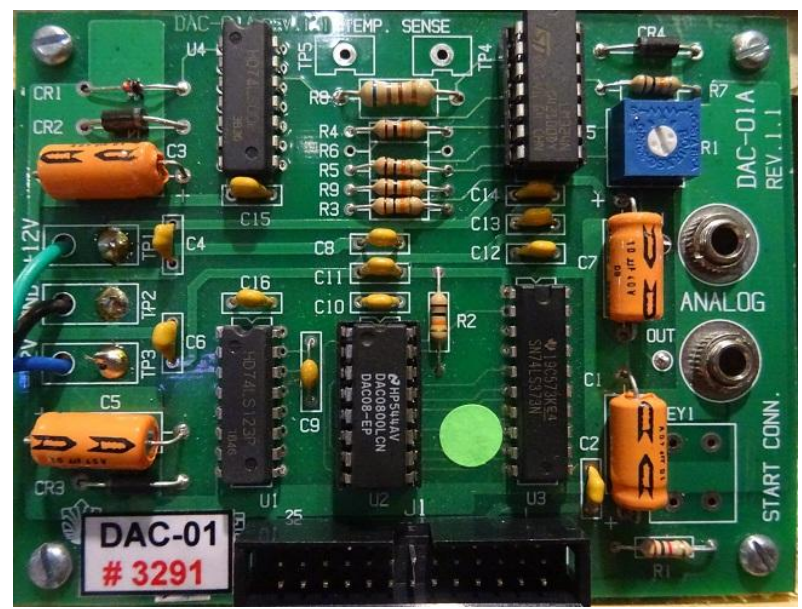

Fig 4: Template Image

\subsection{Result of Canny Edge Detection Algorithm}

Template image is provided to Canny Edge Detection algorithm for converting the image into binary form as described in section 5.2. Fig.5 shows the resulting image after applying canny edge detection algorithm on template image converted into gray scale.

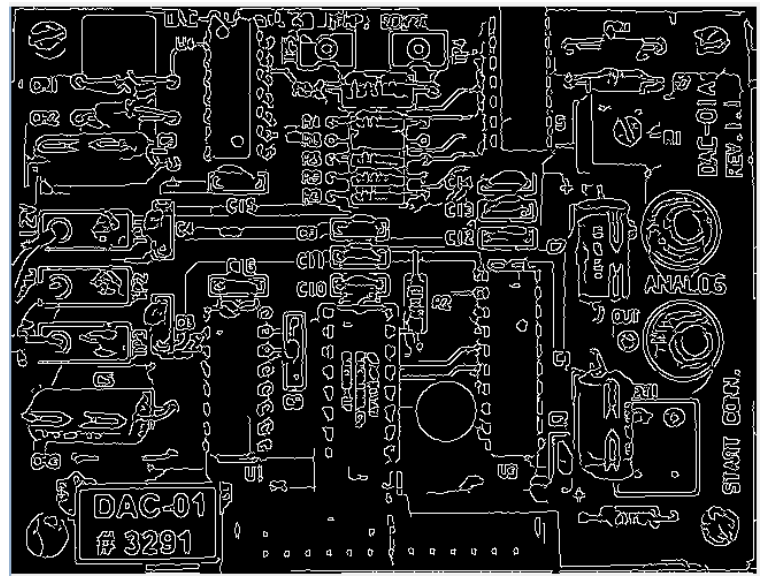

Fig 5: Results of Canny Algorithm

\subsection{XOR Operation}

XOR operation is performed on both the images i.e., template image and reference image to obtain the defects in the sample PCB. Fig.6 shows the results of XOR operation performed on images.

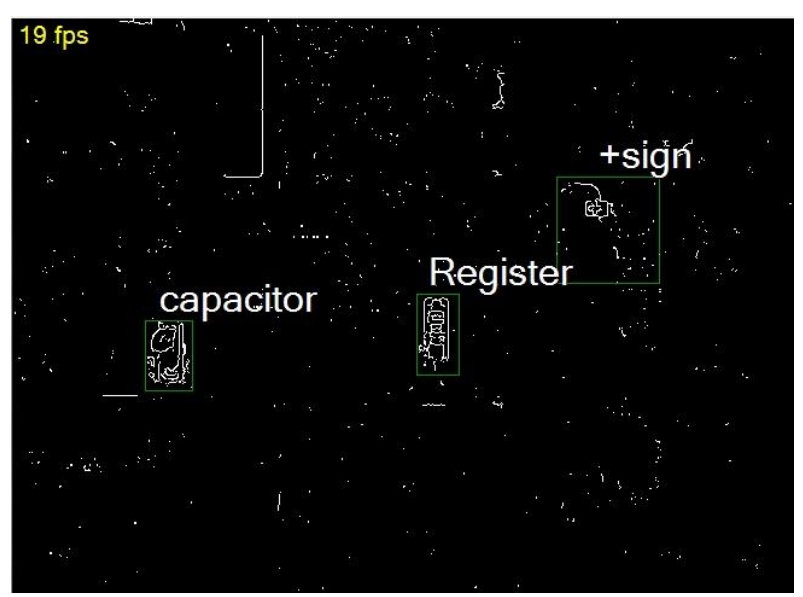

Fig 6: XOR Operation

Obtained XOR results are provided for contour analysis which is used to classify detected faults in various categories. Contour analysis highlights the portion of PCB where fault has occurred and names it as per the type.

\section{CONCLUSION}

The designed system gives the accurate results for PCB testing. It successfully detects whether the sample PCB contains any fault or not. The obtained results are classified according to types of faults in sample PCB. Missing components, polarities, circuit breaks, missing tracks these types of faults are detected and classified accordingly. Reports are generated in terms of occurrence of faults detected in the PCBs. For increasing accuracy of the results, Contour Analysis algorithm is used which results into classification of the faults. 
This system can be improved further as a future scope by using color image processing techniques for making the system more user friendly. Color codes of registers and colors of wires used can be detected further. Conveyor belts can be used to increase speed of image capturing process. System can be improved further by using 3 dimensional images to detect faults such as solder joints and thickness of soldering etc.

\section{REFERENCES}

[1] Sonal Kaushik, Javed Ashraf, "Automated PCB Defect Detection Using Image Subtraction Method", International Journal of Computer Science and Network (IJCSN)Volume 1, Issue 5, www.ijcsn.org ISSN 22775420, October 2012.

[2] Theingi Aye, Aung Soe Khaing, "Automatic Defect Detection and Classification on Printed Circuit Board", International Journal of Societal Applications of Computer Science Vol 3 Issue 3 ISSN 2319 - 8443, March 2014

[3] Xiaojing Tian,Liang Zhao,Huajun Dong, “Application of image processing in the detection of printed circuit board", IEEE Workshop on Electronic Computer And Application,2014.

[4] N. Yogesh Bagrecha, "Quality Control Using Image Processing", et al Int. Journal of Engineering Research and Applications ISSN : 2248-9622, Vol. 4, Issue 3( Version 1),pp.15-18 ,March 2014.

[5] Suhasini A ,Sonal D Kalro, Prathiksha B G, Meghashree B S , Phaneendra H D, "PCB Defect Detection Using
Image Subtraction Algorithm", International Journal of Computer Science Trends and Technology (IJCST) Volume 3 Issue 3, ISSN: 2347-8578 www.ijcstjournal.org, May-June 2015.

[6] "Printed circuit board defect detection using mathematical morphology and MATLAB image processing tools", Researchgate Article, DOI: 10.1109/ICETC.2010.5530052, January 2010.

[7] Takumi Uemura, Gou Koutaki and Keiichi Uchimura, "Image Segmentation based on Edge Detection using Boundry Code", International journal of innovative computing, information and computing, Volume 7, Number 10, October 2011

[8] E. Argyle. "Techniques for edge detection," Proc. IEEE, vol. 59, pp. 285-286, 2012.

[9] F. Bergholm. "Edge focusing," in Proc. 8th Int. Conf. Pattern Recognition, Paris, France, pp. 597- 600, 2013.

[10] Mrs. Namrata. S. Mandvikar, "Augmented Reality Using Contour Analysis In e-learning", International Journal of Innovative Research in Science, Engineering and Technology Vol. 2, Issue 5, May 2013.

[11] Namrata S. Mandvikar1, Sunita Jadhav, "Design and implementation of Augmented Reality learning system using contour analysis", International Journal on Advanced Computer Theory and Engineering (IJACTE), ISSN (print): 2319-2526, Volume -3, Issue -2, 2014. 